\title{
Stability of a Regularized Newton Method with Two Potentials
}

\author{
Boushra Abbas $^{1, *}$, Ramez Koudsieh ${ }^{2}$ \\ ${ }^{1}$ Mathematics Department, Tichreen University, Lattakia, Syria \\ ${ }^{2}$ Mechatroncis Department, Manara University, Lattakia, Syria
}

Email address:

abbas.boushra@yahoo.com (B. Abbas), ramez.koudsieh@manara.edu.sy (R. Koudsieh)

${ }^{*}$ Corresponding author

\section{To cite this article:}

Boushra Abbas, Ramez Koudsieh. Stability of a Regularized Newton Method with Two Potentials. International Journal of Theoretical and Applied Mathematics. Vol. 7, No. 1, 2021, pp. 1-11. doi: 10.11648/j.ijtam.20210701.11

Received: February 14, 2020; Accepted: November 30, 2020; Published: January 22, 2021

\begin{abstract}
In a Hilbert space setting, we introduce dynamical systems, which are linked to Newton and Levenberg-Marquardt methods. They are intended to solve, by splitting methods, inclusions governed by structured monotone operators $M=A+B$, where $A$ is a general maximal monotone operator, and $B$ is monotone and locally Lipschitz continuous. Based on the Minty representation of $A$ as a Lipschitz manifold, we show that these dynamics can be formulated as differential systems, which are relevant to the Cauchy-Lipschitz theorem, and involve separately $B$ and the resolvents of $A$. In the convex subdifferential case, by using Lyapunov asymptotic analysis, we prove a descent minimizing property, and weak convergence to equilibria of the trajectories. Time discretization of these dynamics gives algorithms combining Newton's method and forward-backward methods for solving structured monotone inclusions. The Levenberg-Marquardt regularization term acts in an open loop way. As a byproduct of our study, we can take the regularization coefficient of bounded variation. These stability results are directly related to the study of numerical algorithms that combine forward-backward and Newton's methods.
\end{abstract}

Keywords: Monotone Inclusions, Newton Method, Levenberg-Marquardt Regularization, Dissipative Dynamical Systems, Lyapunov Analysis, Weak Asymptotic Convergence, Forward-Backward Algorithms,

Gradient-Projection Methods

\section{Introduction}

Throughout this paper, $H$ is a real Hilbert space with scalar product $\langle.,$.$\rangle and norm \|\cdot\|$. As a guideline of our study, we use the Newton-like dynamic approach to solving monotone inclusions which was introduced in [7]. To adapt it to structured monotone inclusions and splitting methods, this study was developed in [7], [6] , [2] and [5], where the operator is the sum of the subdifferential of a convex lower semicontinuous function, and the gradient of a convex differentiable function. We wish to extend this study to a non potential case, and so enlarge its range of applications. More precisely, we are going to consider some discrete and continuous Newton-like dynamics, which aim at solving structured monotone inclusions of the following type

$$
\partial \Phi(x)+B x \ni 0
$$

where $\partial \Phi$ is the subdifferential of a convex lower semicontinuous function $\Phi: H \rightarrow \mathbb{R} \cup\{+\infty\}$, and $B$ is a monotone cocoercive operator. Recall that a monotone operator $B: H \rightarrow H$ is cocoercive if there exists a constant $\beta>0$ such that for all $x, y \in H$

$$
\langle B x-B y, x-y\rangle \geq \beta\|B x-B y\|^{2} .
$$

The abstract formulation (1) covers a large variety of problems, see for example [1], [2], [5], [6], [8], [10], [13]. It is directly connected to two important areas, namely convex optimization (take $B=0$ ), and the theory of fixed point for nonexpansive mappings (take $\Phi=0$, and $B=I-T$ with $T$ a nonexpansive mapping). By a classical result, the two operators $\partial \Phi$ and $B$ are maximal monotone, as well as their sum $A=\partial \Phi+B$. We are going to exploit the structure of the maximal monotone operator $A$ in order to develop 
first continuous dynamics, and then, by time discretization, splitting forward-backward algorithms that aim to solve (1). Precisely, our analysis relies on the convergence properties (as $t \rightarrow+\infty)$ of the orbits of the system

$$
\begin{aligned}
& v(t) \in \partial \Phi(x(t)) \\
& \lambda \dot{x}(t)+\dot{v}(t)+v(t)+B(x(t))=0 .
\end{aligned}
$$

In (3), $\lambda$ is a positive constant which acts as a LevenbergMarquard regularization parameter. We make the following standing assumptions: $\varphi$ and $\psi$ are two functions which act on $H$ and satisfy

- $\varphi: H \longrightarrow \mathbb{R} \cup\{+\infty\}$ is convex lower semicontinuous, and proper;

- $\psi: H \longrightarrow \mathbb{R}$ is convex differentiable, and $\nabla \psi$ is Lipschitz continuous on the bounded subsets of $H$.

- $\varphi+\psi$ is bounded from below on $H$.

We are concerned with the study of Newton-like continuous and discrete dynamics attached to solving the the structured minimization problem

$$
(\mathcal{P}) \quad \min \{\varphi(x)+\psi(x): \quad x \in H\} .
$$

Note the asymmetry between $\varphi$, which may be nonsmooth, with extended real values, and $\psi$ which is continuously differentiable, whence the structured property of the above problem. Indeed, we wish to design continuous and discrete dynamics which exploit this particular structure and involve $\varphi$ via implicit operations (like resolvent or proximal operators) and $\psi$ via explicit operations (typically gradient-like methods). So doing we expect obtening new forward-backward splitting methods involving Riemannian metric aspects, and which are close to the Newton method. This approach has been delineated in a series of recent papers, [1], [3], [2], [6], [7]. In this paper we are concerned with the stability properties with respect to the data $\left(\lambda, x_{0}, v_{0}, \ldots\right)$ of the strong solutions of the differential inclusion

$$
\begin{aligned}
& v(t) \in \partial \varphi(x(t)) \\
& \lambda(t) \dot{x}(t)+\dot{v}(t)+v(t)+\nabla \psi(x(t))=0 \\
& x(0)=x_{0}, v(0)=v_{0}
\end{aligned}
$$

Let us now make our standing assumption on function $\lambda(\cdot)$ :

$$
\lambda:[0,+\infty[\longrightarrow] 0,+\infty[
$$

is continuous, and absolutely continuous on each interval

$$
[0, b], 0<b<+\infty \text {. }
$$

Hence $\dot{\lambda}(t)$ exists for almost every $t>0$, and $\dot{\lambda}(\cdot)$ is Lebesgue integrable on each bounded interval $[0, b]$. We stress the fact that we assume $\lambda(t)>0$, for any $t \geq 0$. By continuity of $\lambda(\cdot)$, this implies that, for any $b>0$, there exists some positive finite lower and upper bounds for $\lambda(\cdot)$ on $[0, b]$, i.e., for any $t \in[0, b]$

$$
0<\lambda_{b, \min } \leq \lambda(t) \leq \lambda_{b, \max }<+\infty
$$

Our main interest is to allow $\lambda(t)$ to go to zero as $t \rightarrow$ $+\infty$. This makes the corresponding Levenberg-Marquardt regularization method asymptotically close to the Newton's method.

Let us summarize the results obtained in [2], [12]. Under the above assumptions, for any Cauchy data $x_{0} \in \operatorname{dom} \partial \varphi$ and $v_{0} \in \partial \varphi\left(x_{0}\right)$, there exists a unique strong global solution $(x(\cdot), v(\cdot)):[0,+\infty[\rightarrow H \times H$ of the Cauchy problem (4)-(6). Assuming that the solution set is nonempty, if $\lambda(t)$ tends to zero not too fast, as $t \longrightarrow+\infty$, then $v(t) \longrightarrow 0$ strongly, and $x(t)$ converges weakly to some equilibrium which is a solution of the minimization problem $(\mathcal{P})$. By Minty representation of $\partial \varphi$, the solution pair $(x(\cdot), v(\cdot))$ of (4)-(6) can be represented as follows: set $\mu(t)=\frac{1}{\lambda(t)}$, then for any $t \in[0,+\infty)$,

$$
\begin{aligned}
& x(t)=\operatorname{prox}_{\mu(t) \varphi}(z(t)) ; \\
& v(t)=\nabla \varphi_{\mu(t)}(z(t)),
\end{aligned}
$$

where $z(\cdot):[0,+\infty[\rightarrow H$ is the unique strong global solution of the Cauchy problem

$$
\begin{aligned}
\dot{z}(t) & +(\mu(t)-\dot{\mu}(t)) \nabla \varphi_{\mu(t)}(z(t)) \\
& +\mu(t) \nabla \psi\left(\operatorname{prox}_{\mu(t) \Phi}(z(t))\right) \\
& =0 \\
z(0) & =x_{0}+\mu(0) v_{0} .
\end{aligned}
$$

Let us recall that prox $_{\mu \varphi}$ is the proximal mapping associated to $\mu \varphi$. Equivalently, $\operatorname{prox}_{\mu \varphi}=(I+\mu \partial \phi)^{-1}$ is the resolvent of index $\mu>0$ of the maximal monotone operator $\partial \varphi$, and $\nabla \varphi_{\mu}$ is its Yosida approximation of index $\mu>0$.

Let us stress the fact that, for each $t>0$, the operators $\operatorname{prox}_{\mu(t) \varphi}: H \longrightarrow H, \nabla \varphi_{\mu(t)}: H \longrightarrow H$ are everywhere defined and Lipschitz continuous, which makes this system relevant to the Cauchy-Lipschitz theorem in the nonautonomous case, which naturally suggests good stability results of the solution of (4)-(6) with respect to the data.

This paper is organized as follows: We first establish a priori energy estimates on the trajectories. Then we consider the case where $\lambda$ is locally absolutely continuous. Note that it is important, for numerical reasons, to study the stability of the solution with respect to perturbations of the data, and in particular of $\lambda$ which plays a crucial role in the regularization process. In Theorem 3.1 we prove the Lipschitz continuous dependence of the solution with respect to $\lambda$. Moreover, the Lipschitz constant only depends on the $L^{1}$ norm of the time derivative of $\lambda$. Finally, we extend our analysis to the case where $\lambda$ is a function with bounded variation (possibly involving jumps). We use a regularization by convolution method in order to reduce to the smooth case, and then pass to the limit in the equations. So doing, in Theorem 4.1 and Corollary 4.1, we prove the existence and uniqueness of a strong solution for (4)-(6), in the case where $\lambda$ is a function with bounded variation. 


\section{A Priori Estimates}

The linear space $H \times H$ is equipped with its usual Hilbertian norm $\|(\xi, \zeta)\|=\sqrt{\|\xi\|^{2}+\|\zeta\|^{2}}$. In this section we work on a fixed bounded interval $[0, T]$, and following assumption (8), we suppose that there exists some positive constant $c_{0}$ such that

$$
0<c_{0} \leq \lambda(t) \text { for all } t \in[0, T]
$$

We will also assume that $\nabla \psi$ is $L_{\psi}$-Lipschitz continuous. Indeed, this is not a restrictive assumption since one can reduce the study to trajectories belonging to a fixed ball in $H$. We will often omit the time variable $t$ and write $x, v \ldots$ for $x(t), v(t) \ldots$. when no ambiguity arises.

In the following two Propositions we denote by $(x(\cdot), v(\cdot)):[0,+\infty) \rightarrow H \times H$ the unique strong global solution of the Cauchy problem (4)-(6).

Proposition 2.1. Let $(x, v)$ be the strong solution of system (4)-(6) on $[0, T], T>0$. Then

$$
\begin{aligned}
& \int_{0}^{T}\|\dot{x}(t)\|^{2} \leq \frac{1}{c_{0}}\left((\varphi+\psi)\left(x_{0}\right)-\inf _{H}(\varphi+\psi)\right) . \\
& \|x\|_{L^{\infty}(0, T ; H)} \leq\left\|x_{0}\right\|+\sqrt{\frac{T}{c_{0}}}\left((\varphi+\psi)\left(x_{0}\right)-\inf _{H}(\varphi+\psi)\right)^{\frac{1}{2}} .
\end{aligned}
$$

Proof. a) For almost every $t>0, \dot{x}(t)$ and $\dot{v}(t)$ are well defined, thus

$$
\langle\dot{x}(t), \dot{v}(t)\rangle=\lim _{h \rightarrow 0} \frac{1}{h^{2}}\langle x(t+h)-x(t), v(t+h)-v(t)\rangle .
$$

By equation (4), we have $v(t) \in \partial \varphi(x(t))$.

Since $\partial \varphi: H \rightrightarrows H$ is monotone

$$
\langle x(t+h)-x(t), v(t+h)-v(t)\rangle \geq 0 .
$$

Dividing by $h^{2}$ and passing to the limit preserves the inequality, which yields

$$
\langle\dot{x}(t), \dot{v}(t)\rangle \geq 0 \text {. }
$$

By taking the inner product of both sides of (5) by $\dot{x}(t)$ we obtain

$\lambda(t)\|\dot{x}(t)\|^{2}+\langle\dot{x}(t), \dot{v}(t)\rangle+\langle v(t), \dot{x}(t)\rangle+\langle\nabla \psi(x(t)), \dot{x}(t)\rangle=0$.

By (17) we infer

$$
\lambda(t)\|\dot{x}(t)\|^{2}+\langle v(t), \dot{x}(t)\rangle+\langle\nabla \psi(x(t)), \dot{x}(t)\rangle \leq 0 .
$$

Noticing that $x$ and $v$ are continuous on $[0, T]$, hence bounded, and that $\lambda$ is bounded from below on $[0, T]$ by a positive number, one easily gets from (18) that

$$
\dot{x} \in L^{2}(0, T ; H) \text {. }
$$

For our stability analysis, we now establish a precise estimate of the $L^{2}$ norm of $\dot{x}$. By the classical derivation chain rule

$$
\frac{d}{d t} \psi(x(t))=\langle\nabla \psi(x(t)), \dot{x}(t)\rangle .
$$

We appeal to a similar formula which is still valid for a convex lower semicontinuous proper function $\varphi: H \rightarrow$ $\mathbb{R} \cup\{+\infty\}$. Notice that

i) $v(t) \in \partial \varphi(x(t))$ for almost every $t \in[0, T]$;

ii) $v$ is continuous on $[0, T]$, and hence belongs to $L^{2}(0, T ; H)$;

iii) $\dot{x} \in L^{2}(0, T ; H)$ by (19).
By $i), i i), i i i)$, conditions of [11, Lemma 3.3] are satisfied, which allows to deduce that $t \mapsto \varphi(x(t))$ is absolutely continuous on $[0, T]$, and for almost $t \in[0, T]$,

$$
\frac{d}{d t} \varphi(x(t))=\langle v(t), \dot{x}(t)\rangle \text {. }
$$

Combining (18) with (20) and (21) we obtain

$$
\lambda(t)\|\dot{x}(t)\|^{2}+\frac{d}{d t}(\varphi(x(t))+\psi(x(t))) \leq 0 .
$$

By integrating the above inequality from 0 to $T$ we obtain

$$
\int_{0}^{T} \lambda(t)\|\dot{x}(t)\|^{2} d t+(\varphi+\psi)(x(T)) \leq(\varphi+\psi)(x(0)) .
$$

Since $\varphi+\psi$ is bounded from below on $H$, and $\lambda$ is minorized by the positive constant $c_{0}$ on $[0, T]$ (see (14)), we infer

$$
\int_{0}^{T}\|\dot{x}(t)\|^{2} d t \leq \frac{1}{c_{0}}\left((\varphi+\psi)\left(x_{0}\right)-\inf _{H}(\varphi+\psi)\right) .
$$

b) Since $x(\cdot)$ is absolutely continuous on bounded sets, for any $t \in[0, T]$

$$
x(t)=x_{0}+\int_{0}^{t} \dot{x}(\tau) d \tau .
$$

Passing to the norm, and using Cauchy-Schwarz inequality yields

$$
\begin{aligned}
\|x(t)\| & \leq\left\|x_{0}\right\|+\int_{0}^{t}\|\dot{x}(\tau)\| d \tau \\
& \leq\left\|x_{0}\right\|+\sqrt{T}\left(\int_{0}^{T}\|\dot{x}(\tau)\|^{2} d \tau\right)^{\frac{1}{2}} .
\end{aligned}
$$

Combining the above inequality with (24) gives

$$
\|x(t)\| \leq\left\|x_{0}\right\|+\sqrt{\frac{T}{c_{0}}}\left((\varphi+\psi)\left(x_{0}\right)-\inf _{H}(\varphi+\psi)\right)^{\frac{1}{2}} .
$$


This being true for any $t \in[0, T]$

$$
\|x\|_{L^{\infty}(0, T ; H)} \leq \mid x_{0} \|+\sqrt{\frac{T}{c_{0}}}\left((\varphi+\psi)\left(x_{0}\right)-\inf _{H}(\varphi+\psi)\right)^{\frac{1}{2}} .
$$

Let us now exploit another a priori energy estimate.

Proposition 2.2. Let $(x, v)$ be the strong solution of system (4)-(6) on $[0, T], T>0$. Then

$$
\begin{aligned}
& \int_{0}^{T}\|\dot{v}(t)\|^{2} d t \leq\left\|v_{0}\right\|^{2}+2 T\left\|\nabla \psi\left(x_{0}\right)\right\|^{2}+\frac{2 T^{2} L_{\psi}^{2}}{c_{0}}\left((\varphi+\psi)\left(x_{0}\right)-\inf _{H}(\varphi+\psi)\right) \\
& \|v\|_{L^{\infty}(0, T ; H)} \leq\left\|v_{0}\right\|+\sqrt{2 T}\left\|\nabla \psi\left(x_{0}\right)\right\|+\sqrt{\frac{2}{c_{0}}} T L_{\psi}\left((\varphi+\psi)\left(x_{0}\right)-\inf _{H}(\varphi+\psi)\right)^{\frac{1}{2}} .
\end{aligned}
$$

Proof. By taking the scalar product of (5) by $\dot{v}(t)$ we obtain

$$
\lambda(t)\langle\dot{x}(t), \dot{v}(t)\rangle+\|\dot{v}(t)\|^{2}+\langle v(t), \dot{v}(t)\rangle+\langle\nabla \psi(x(t)), \dot{v}(t)\rangle=0 .
$$

By (17) we infer

$$
\|\dot{v}(t)\|^{2}+\langle v(t), \dot{v}(t)\rangle+\langle\nabla \psi(x(t)), \dot{v}(t)\rangle \leq 0
$$

Hence

$$
\|\dot{v}(t)\|^{2}+\frac{1}{2} \frac{d}{d t}\|v(t)\|^{2} \leq\|\nabla \psi(x(t))\|\|\dot{v}(t)\| \leq \frac{1}{2}\|\nabla \psi(x(t))\|^{2}+\frac{1}{2}\|\dot{v}(t)\|^{2}
$$

which implies

$$
\|\dot{v}(t)\|^{2}+\frac{d}{d t}\|v(t)\|^{2} \leq\|\nabla \psi(x(t))\|^{2} .
$$

By integrating the above inequality we deduce that, for any $t \in[0, T]$

$$
\int_{0}^{t}\|\dot{v}(\tau)\|^{2} d \tau+\|v(t)\|^{2} \leq\left\|v_{0}\right\|^{2}+\int_{0}^{T}\|\nabla \psi(x(\tau))\|^{2} d \tau .
$$

Since $\nabla \psi$ is $L_{\psi}$-Lipschitz continuous

$$
\|\nabla \psi(x(\tau))\| \leq\left\|\nabla \psi\left(x_{0}\right)\right\|+L_{\psi}\left\|x(\tau)-x_{0}\right\| .
$$

A careful look at the proof of (28) gives the more precise estimate

$$
\left\|x-x_{0}\right\|_{L^{\infty}(0, T ; H)} \leq \sqrt{\frac{T}{c_{0}}}\left((\varphi+\psi)\left(x_{0}\right)-\inf _{H}(\varphi+\psi)\right)^{\frac{1}{2}}
$$

Hence for any $\tau \in[0, T]$

$$
\|\nabla \psi(x(\tau))\| \leq\left\|\nabla \psi\left(x_{0}\right)\right\|+L_{\psi} \sqrt{\frac{T}{c_{0}}}\left((\varphi+\psi)\left(x_{0}\right)-\inf _{H}(\varphi+\psi)\right)^{\frac{1}{2}} .
$$

Combining (34) with (37) gives

$$
\int_{0}^{t}\|\dot{v}(\tau)\|^{2} d \tau+\|v(t)\|^{2} \leq\left\|v_{0}\right\|^{2}+2 T\left\|\nabla \psi\left(x_{0}\right)\right\|^{2}+\frac{2 T^{2} L_{\psi}^{2}}{c_{0}}\left((\varphi+\psi)\left(x_{0}\right)-\inf _{H}(\varphi+\psi)\right) .
$$

As a consequence

$$
\int_{0}^{T}\|\dot{v}(t)\|^{2} d t \leq\left\|v_{0}\right\|^{2}+2 T\left\|\nabla \psi\left(x_{0}\right)\right\|^{2}+\frac{2 T^{2} L_{\psi}^{2}}{c_{0}}\left((\varphi+\psi)\left(x_{0}\right)-\inf _{H}(\varphi+\psi)\right),
$$

and

$$
\|v\|_{L^{\infty}(0, T ; H)} \leq\left\|v_{0}\right\|+\sqrt{2 T}\left\|\nabla \psi\left(x_{0}\right)\right\|+\sqrt{\frac{2}{c_{0}}} T L_{\psi}\left((\varphi+\psi)\left(x_{0}\right)-\inf _{H}(\varphi+\psi)\right)^{\frac{1}{2}} .
$$

which ends the proof. 
We can now deduce from the two preceding propositions an a priori bound on the $L^{\infty}$ norm of $\dot{x}$ and $\dot{v}$.

$$
\begin{aligned}
\|\dot{x}\|_{L^{\infty}(0, T ; H)} & \leq \frac{\left\|v_{0}\right\|}{c_{0}}+\frac{1+\sqrt{2 T}}{c_{0}}\left\|\nabla \psi\left(x_{0}\right)\right\|+\frac{(\sqrt{2} T+\sqrt{T}) L_{\psi}}{c_{0}^{\frac{3}{2}}}\left((\varphi+\psi)\left(x_{0}\right)-\inf _{H}(\varphi+\psi)\right)^{\frac{1}{2}} \\
\|\dot{v}\|_{L^{\infty}(0, T ; H)} & \leq\left\|v_{0}\right\|+(1+\sqrt{2 T})\left\|\nabla \psi\left(x_{0}\right)\right\|+\frac{(\sqrt{2} T+\sqrt{T}) L_{\psi}}{c_{0}^{\frac{1}{2}}}\left((\varphi+\psi)\left(x_{0}\right)-\inf _{H}(\varphi+\psi)\right)^{\frac{1}{2}}
\end{aligned}
$$

Proof. a) Let us return to the equation obtained by taking the inner product of both sides of (5) by $\dot{x}(t)$

$$
\lambda(t)\|\dot{x}(t)\|^{2}+\langle\dot{x}(t), \dot{v}(t)\rangle+\langle v(t), \dot{x}(t)\rangle+\langle\nabla \psi(x(t)), \dot{x}(t)\rangle=0 .
$$

By (17), and $\lambda$ is minorized by the positive constant $c_{0}$ on $[0, T]$,we infer

$$
c_{0}\|\dot{x}(t)\|^{2}+\langle v(t)+\nabla \psi(x(t)), \dot{x}(t)\rangle \leq 0 .
$$

Hence

$$
\|\dot{x}(t)\| \leq \frac{1}{c_{0}}\|v(t)+\nabla \psi(x(t))\| \leq \frac{1}{c_{0}}\left(\|v(t)\|+\left\|\nabla \psi\left(x_{0}\right)\right\|+L_{\psi}\left\|x(t)-x_{0}\right\|\right) .
$$

By (36) and (40) we deduce that

$$
\begin{aligned}
\|\dot{x}\|_{L^{\infty}(0, T ; H)} & \leq \frac{1}{c_{0}}\left(\left\|v_{0}\right\|+\sqrt{2 T}\left\|\nabla \psi\left(x_{0}\right)\right\|+\sqrt{\frac{2}{c_{0}}} T L_{\psi}\left((\varphi+\psi)\left(x_{0}\right)-\inf _{H}(\varphi+\psi)\right)^{\frac{1}{2}}\right) \\
& +\frac{1}{c_{0}}\left\|\nabla \psi\left(x_{0}\right)\right\|+\frac{L_{\psi}}{c_{0}} \sqrt{\frac{T}{c_{0}}}\left((\varphi+\psi)\left(x_{0}\right)-\inf _{H}(\varphi+\psi)\right)^{\frac{1}{2}} \\
& \leq \frac{\left\|v_{0}\right\|}{c_{0}}+\frac{1+\sqrt{2 T}}{c_{0}}\left\|\nabla \psi\left(x_{0}\right)\right\|+\frac{(\sqrt{2} T+\sqrt{T}) L_{\psi}}{c_{0}^{\frac{3}{2}}}\left((\varphi+\psi)\left(x_{0}\right)-\inf _{H}(\varphi+\psi)\right)^{\frac{1}{2}} .
\end{aligned}
$$

b) Let us return to the equation obtained by taking the inner product of both sides of (5) by $\dot{v}(t)$

$$
\lambda(t)\langle\dot{x}(t), \dot{v}(t)\rangle+\|\dot{v}(t)\|^{2}+\langle v(t), \dot{v}(t)\rangle+\langle v \nabla \psi(x(t)), \dot{v}(t)\rangle=0 .
$$

A similar argument as above yields

$$
\|\dot{v}(t)\| \leq\|v(t)+\nabla \psi(x(t))\|
$$

from which we deduce the result.

Let us enunciate some straight consequences of Proposition 2.3.

Corollary 2.1. The following properties hold: for any $0<T<+\infty$

1. $t \mapsto x(t)$ is Lipschitz continuous on $[0, T]$ with constant

$$
\frac{\left\|v_{0}\right\|}{c_{0}}+\frac{1+\sqrt{2 T}}{c_{0}}\left\|\nabla \psi\left(x_{0}\right)\right\|+\frac{(\sqrt{2} T+\sqrt{T}) L_{\psi}}{c_{0}^{\frac{3}{2}}}\left((\varphi+\psi)\left(x_{0}\right)-\inf _{H}(\varphi+\psi)\right)^{\frac{1}{2}}
$$

2. $t \mapsto v(t)$ is Lipschitz continuous on $[0, T]$, with constant

$$
\left\|v_{0}\right\|+(1+\sqrt{2 T})\left\|\nabla \psi\left(x_{0}\right)\right\|+\frac{(\sqrt{2} T+\sqrt{T}) L_{\psi}}{c_{0}^{\frac{1}{2}}}\left((\varphi+\psi)\left(x_{0}\right)-\inf _{H}(\varphi+\psi)\right)^{\frac{1}{2}}
$$

\section{Stability Results}

In the next theorem we analyze the Lipschitz continuous dependence of the solution $(x, v)$ of the Cauchy problem (4)-(6) with respect to the function $\lambda$ and the initial point $\left(x_{0}, v_{0}\right)$. Our demonstration is based on that followed in [6],[9],[14].

Theorem 3.1. Suppose that $\lambda, \eta:[0, T] \longrightarrow\left[c_{0},+\infty\left[\right.\right.$ are absolutely continuous functions, with $T>0$ and $c_{0}>0$. Let 
$(x, v),(y, w):[0, T] \longrightarrow H \times H$ be the respective strong solutions of the inclusions

$$
\begin{aligned}
& \lambda \dot{x}+\dot{v}+v+\nabla \psi(x)=0, v \in \partial \varphi(x), x(0)=x_{0}, v(0)=v_{0}, \\
& \eta \dot{y}+\dot{w}+w+\nabla \psi(y)=0, w \in \partial \varphi(y), y(0)=y_{0}, w(0)=w_{0} .
\end{aligned}
$$

Define $\theta:[0, T] \longrightarrow \mathbb{R}$, for each $t \in[0, T]$, by

$$
\theta(t)=\sqrt{c_{0}^{2}\|x(t)-y(t)\|^{2}+\|v(t)-w(t)\|^{2}} .
$$

Then

$$
\|\theta\|_{L^{\infty}([0, T])} \leq\left[\frac{\lambda(0)+\eta(0)}{2}\left\|x_{0}-y_{0}\right\|+\left\|v_{0}-w_{0}\right\|+\frac{C}{2}\|\lambda-\eta\|_{L^{1}([0, T])}\right] \times \exp \left(\frac{\|\dot{\lambda}+\dot{\eta}\|_{L^{1}}}{2 c_{0}}+T\left(1+\frac{L_{\psi}}{c_{0}}\right)\right)
$$

with

$$
\begin{aligned}
& C=\frac{\left\|v_{0}\right\|+\left\|w_{0}\right\|}{c_{0}}+\frac{1+\sqrt{2 T}}{c_{0}}\left(\left\|\nabla \psi\left(x_{0}\right)\right\|+\left\|\nabla \psi\left(y_{0}\right)\right\|\right)+ \\
& \quad \frac{(\sqrt{2} T+\sqrt{T}) L_{\psi}}{c_{0}^{\frac{3}{2}}}\left(\left((\varphi+\psi)\left(x_{0}\right)-\inf _{H}(\varphi+\psi)\right)^{\frac{1}{2}}+\left((\varphi+\psi)\left(y_{0}\right)-\inf _{H}(\varphi+\psi)\right)^{\frac{1}{2}}\right) .
\end{aligned}
$$

In particular, if $x_{0}=y_{0}, v_{0}=w_{0}$, then

$$
\|\theta\|_{L^{\infty}([0, T])} \leq \frac{C}{2}\|\lambda-\eta\|_{L^{1}([0, T])} \times \exp \left(\frac{\|\dot{\lambda}+\dot{\eta}\|_{L^{1}([0, T])}}{2 c_{0}}+T\left(1+\frac{L_{\psi}}{c_{0}}\right)\right),
$$

with

$$
C=\frac{2\left\|v_{0}\right\|}{c_{0}}+2 \frac{1+\sqrt{2 T}}{c_{0}}\left\|\nabla \psi\left(x_{0}\right)\right\|+2 \frac{(\sqrt{2} T+\sqrt{T}) L_{\psi}}{c_{0}^{\frac{3}{2}}}\left((\varphi+\psi)\left(x_{0}\right)-\inf _{H}(\varphi+\psi)\right)^{\frac{1}{2}} .
$$

Proof. To simplify the exposition, define $\gamma:[0, T] \longrightarrow \mathbb{R}$

$$
\gamma=\frac{\lambda+\eta}{2}
$$

Using the assumptions $\lambda, \eta \geq c_{0}$ and the monotonicity of $\partial \varphi$ we conclude that for any $t \in[0, T]$

$$
c_{0} \leq \gamma,\langle x-y, v-w\rangle \geq 0 \text {. }
$$

Therefore

$$
\theta \leq \sqrt{\gamma^{2}\|x-y\|^{2}+\|v-w\|^{2}} \leq\|\gamma(x-y)+(v-w)\| .
$$

Let us show that $\theta$ satisfies a Gronwall inequality. Let us start from

$$
\frac{d}{d t}[\gamma(x-y)+(v-w)]=\dot{\gamma}(x-y)+\gamma(\dot{x}-\dot{y})+(\dot{v}-\dot{w}) .
$$

In view of (43) and (44),

$$
(\dot{v}-\dot{w})=-\lambda \dot{x}+\eta \dot{y}-(v-w)-(\nabla \psi(x)-\nabla \psi(y)) .
$$

Combining the two above relations gives

$$
\begin{aligned}
\frac{d}{d t}[\gamma(x-y)+(v-w)] & =\dot{\gamma}(x-y)+\gamma(\dot{x}-\dot{y})-\lambda \dot{x}+\eta \dot{y}-(v-w)-(\nabla \psi(x)-\nabla \psi(y)), \\
& =\dot{\gamma}(x-y)+\frac{\eta-\lambda}{2}(\dot{x}+\dot{y})-(v-w)-(\nabla \psi(x)-\nabla \psi(y)) .
\end{aligned}
$$

Since $\gamma, x, y, w, v$ are absolutely continuous, the function $\gamma(x-y)+(v-w)$ is also absolutely continuous. As a consequence, 
integration of the above inequality on $[0, s]$, for $s \in[0, T]$, yields

$$
\begin{aligned}
& {[\gamma(x-y)+(v-w)](s)-[\gamma(x-y)+(v-w)](0)=} \\
& \frac{1}{2} \int_{0}^{s}(\eta-\lambda)(\dot{x}+\dot{y}) d t+\int_{0}^{s}(\dot{\gamma}(x-y)-(v-w)) d t-\int_{0}^{s}(\nabla \psi(x)-\nabla \psi(y)) d t .
\end{aligned}
$$

Passing to the norm

$$
\begin{aligned}
& \|[\gamma(x-y)+(v-w)](s)\| \leq \gamma(0)\left\|x_{0}-y_{0}\right\|+\left\|v_{0}-w_{0}\right\| \\
& \quad+\frac{1}{2} \int_{0}^{s}|\lambda-\eta|\|\dot{x}+\dot{y}\| d t+\int_{0}^{s}\|\dot{\gamma}(x-y)-(v-w)\| d t+\int_{0}^{s}\|\nabla \psi(x)-\nabla \psi(y)\| d t .
\end{aligned}
$$

By Lipschitz continuity property of $\nabla \psi$, and definition of $\theta$, we have

$$
\|\nabla \psi(x(t))-\nabla \psi(y(t))\| \leq L_{\psi}\|x(t)-y(t)\| \leq \frac{L_{\psi}}{c_{0}} \theta(t) .
$$

On the other hand

$$
\begin{aligned}
\|\dot{\gamma}(x-y)-(v-w)\|^{2} & =|\dot{\gamma}|^{2}\|x-y\|^{2}+\|v-w\|^{2}+2\left\langle c_{0}(x-y), \frac{\dot{\gamma}}{c_{0}}(w-v)\right\rangle \\
& \leq|\dot{\gamma}|^{2}\|x-y\|^{2}+\|v-w\|^{2}+c_{0}^{2}\|x-y\|^{2}+\frac{|\dot{\gamma}|^{2}}{c_{0}^{2}}\|v-w\|^{2} \\
& =\left(\frac{|\dot{\gamma}|^{2}}{c_{0}^{2}}+1\right) \theta^{2},
\end{aligned}
$$

Combining (48), (49), (50) and $\theta \leq\|\gamma(x-y)+(v-w)\|$, we obtain

$$
\begin{aligned}
\theta(s) & \leq \gamma(0)\left\|x_{0}-y_{0}\right\|+\left\|v_{0}-w_{0}\right\|+\frac{1}{2} \int_{0}^{s}|\lambda-\eta|\|\dot{x}+\dot{y}\| d t+\int_{0}^{s} \theta(t)\left[\sqrt{\frac{|\dot{\gamma}|^{2}}{c_{0}^{2}}+1}+\frac{L_{\psi}}{c_{0}}\right] d t \\
& \leq \gamma(0)\left\|x_{0}-y_{0}\right\|+\left\|v_{0}-w_{0}\right\|+\frac{1}{2}\|\lambda-\eta\|_{L^{1}([0, T])}\|\dot{x}+\dot{y}\|_{L^{\infty}([0, T])}+\int_{0}^{s} \theta(t)\left[\frac{|\dot{\gamma}|}{c_{0}}+1+\frac{L_{\psi}}{c_{0}}\right] d t .
\end{aligned}
$$

Applying Gronwall's inequality yields

$$
\theta(s) \leq\left[\gamma(0)\left\|x_{0}-y_{0}\right\|+\left\|v_{0}-w_{0}\right\|+\frac{1}{2}\|\lambda-\eta\|_{L^{1}([0, T])}\|\dot{x}+\dot{y}\|_{L^{\infty}([0, T])}\right] \times \exp \int_{0}^{s}\left[\frac{|\dot{\gamma}|}{c_{0}}+1+\frac{L_{\psi}}{c_{0}}\right] d t
$$

Combining this estimation with the bound on the $L^{\infty}$ norm of $\dot{x}$ and $\dot{y}$ (see Proposition 2.3)) gives

$$
\|\theta\|_{L^{\infty}([0, T])} \leq\left[\gamma(0)\left\|x_{0}-y_{0}\right\|+\left\|v_{0}-w_{0}\right\|+\frac{C}{2}\|\lambda-\eta\|_{L^{1}([0, T])}\right] \times \exp \int_{0}^{T}\left[\frac{|\dot{\gamma}|}{c_{0}}+1+\frac{L_{\psi}}{c_{0}}\right] d t
$$

with

$$
\begin{aligned}
C & =\frac{\left\|v_{0}\right\|+\left\|w_{0}\right\|}{c_{0}}+\frac{1+\sqrt{2 T}}{c_{0}}\left(\left\|\nabla \psi\left(x_{0}\right)\right\|+\left\|\nabla \psi\left(y_{0}\right)\right\|\right) \\
& +\frac{(\sqrt{2} T+\sqrt{T}) L_{\psi}}{c_{0}^{\frac{3}{2}}}\left(\left((\varphi+\psi)\left(x_{0}\right)-\inf _{H}(\varphi+\psi)\right)^{\frac{1}{2}}+\left((\varphi+\psi)\left(y_{0}\right)-\inf _{H}(\varphi+\psi)\right)^{\frac{1}{2}}\right) .
\end{aligned}
$$

Equivalently

$$
\|\theta\|_{L^{\infty}([0, T])} \leq\left[\frac{\lambda(0)+\eta(0)}{2}\left\|x_{0}-y_{0}\right\|+\left\|v_{0}-w_{0}\right\|+\frac{C}{2}\|\lambda-\eta\|_{L^{1}([0, T])}\right] \times \exp \left(\frac{\|\dot{\lambda}+\dot{\eta}\|_{L^{1}([0, T])}}{2 c_{0}}+T\left(1+\frac{L_{\psi}}{c_{0}}\right)\right)
$$


Remark 3.1. In the cas $\psi=0$ we recover exactly the same stability results as [6, Theorem 3.1].

Remark 3.1. - It is worth noticing that, besides the Lipschitz continuous dependence with respect to $\lambda$ of the solution $(x, v)$ of (4)-(6), Theorem (3.1) also provides its continuous dependence with respect to the initial data $\left(x_{0}, v_{0}\right)$. More precisely, if $\left(x_{n}, v_{n}\right)$ (resp. $\left.(x, v)\right)$ is the solution of (4)-(6) corresponding to the Cauchy data $\left(x_{0 n}, v_{0 n}\right)$ (resp. $\left.\left(x_{0}, v_{0}\right)\right)$, as a direct consequence of (45) we obtain for all $T>0,\left(\partial \varphi \ni\left(x_{0 n}, v_{0 n}\right) \longrightarrow\left(x_{0}, v_{0}\right)\right) \Longrightarrow\left(x_{n}, v_{n}\right) \longrightarrow$ $(x, v)$ uniformly on $[0, T]$.

Note also that, by contrast with semigroup generated by a subdifferential convex [11],[15], there is no regularizing effect on the initial condition: there is no way to define a solution of (4)-(6) for $x_{0} \in \overline{\operatorname{dom} \partial \varphi} \backslash \operatorname{dom} \partial \varphi$ since in that case, for any approximation sequence $x_{0 n} \in \operatorname{dom} \partial \varphi$ and $v_{0 n} \in \partial \varphi\left(x_{0 n}\right)$, one has $\lim _{n}\left\|v_{0 n}\right\|=+\infty$, which would imply the blow-up of the sequence $\left(x_{n}, v_{n}\right)$ as $n \longrightarrow+\infty$, on any finite interval.

Remark 3.2. Another approach is to study the equivalent problem (13)-(13), based on the known stability results for the Cauchy-Lipschitz problem. Although conceptually simple, this approach seems more technical.

\section{Bounded Variation Regularization Coefficient $\lambda(\cdot)$}

Let us suppose that $\lambda(\cdot):[0, T] \longrightarrow] 0, \infty[$ is of bounded variation on $[0, T]$, where $T>0$. That is $\operatorname{TV}(\lambda,[0, T])<$ $+\infty$, where $\operatorname{TV}(\lambda,[0, T])$ is the total variation of $\lambda$ on $[0, T]$ :

$$
\operatorname{TV}(\lambda,[0, T])=\sup \sum_{i=1}^{p}\left|\lambda\left(\tau_{i}\right)-\lambda\left(\tau_{i-1}\right)\right|,
$$

the supremum being taken over all $p \in \mathbb{N}$ and all strictly increasing sequences $\tau_{0}<\tau_{1}<\cdots<\tau_{p}$ of points of $[0, T]$. Function $\lambda$ may involve jumps. We also suppose that $\lambda$ is bounded away from 0 :

$$
\inf \lambda([0, T])>0 \text {. }
$$

The following lemmas which are proved in [6] gather some classical facts concerning the approximation of functions of bounded variation by smooth functions together with some technical results useful for sequel.

Lemma 4.1. Let $\lambda:[0, T] \longrightarrow] 0, \infty[$ be of bounded variation on $[0, T]$. Then there exists a sequence $\left(\lambda_{n}\right)_{n \in \mathbb{N}}$, with $\lambda_{n} \in C^{\infty}([0, T])$ for each $n \in \mathbb{N}$, such that

i) $\inf \lambda([0, T]) \leq \lambda_{n}(t) \leq \sup \lambda([0, T]), \forall t \in[0, T]$, $\forall n \in \mathbb{N}$.

In particular, $\lambda_{n} \geq 0$ if $\lambda \geq 0$;

ii) $\lambda_{n} \longrightarrow \lambda$ in $L^{p}(0, T)$ for any $1 \leq p<\infty$;

iii) $\operatorname{TV}\left(\lambda_{n},[0, T]\right)=\int_{0}^{T}\left|\dot{\lambda}_{n}(t)\right| d t \leq \operatorname{TV}(\lambda,[0, T])$.

Lemma 4.2. Let $z_{n}, z \in C([0, T], H)$ be such that $z_{n} \longrightarrow$ $z$ uniformly and $\left(z_{n}\right)_{n}$ is L-Lipschitz continuous for some positive constant $L$ independent of $n \in \mathbb{N}$. Let $\lambda_{n} \longrightarrow$ $\lambda$ in $L^{2}(0, T)$. Then $\lambda_{n} \dot{z}_{n}$ converges weakly to $\lambda \dot{z}$ in $L^{2}(0, T ; H)$.

We can now state the main result of this section.

Theorem 4.1. Let $\lambda:[0, T] \longrightarrow] 0, \infty[$ be of bounded variation on $[0, T]$, and suppose $c_{0}=\inf \lambda([0, T])>0$. Let $x_{0} \in \operatorname{dom} \partial \varphi$ and $v_{0} \in \partial \varphi\left(x_{0}\right), \quad v_{0} \neq 0$. Then there is existence and uniqueness of a strong solution $(x, v)$ : $[0, T] \longrightarrow H \times H$ of the Cauchy problem

$$
\begin{aligned}
& v(t) \in \partial \varphi(x(t)), \quad 0 \leq t \leq T \\
& \lambda(t) \dot{x}(t)+\dot{v}(t)+v(t)+\nabla \psi(x(t))=0, \\
& \text { a.e. } 0 \leq t \leq T \\
& x(0)=x_{0}, \quad v(0)=v_{0} .
\end{aligned}
$$

Proof. Existence: According to Lemma 4.1, there exists a sequence of functions $\left(\lambda_{n}\right)_{n \in \mathbb{N}}$ in $C^{\infty}([0, T])$, with $\lambda_{n}(t) \geq$ $c_{0}$, which converges to $\lambda$ in $L^{p}(0, T)$ for $p \geq 1$, and satisfies the condition

$$
\int_{0}^{T}\left|\dot{\lambda}_{n}(t)\right| d t \leq \mathrm{TV}(\lambda)
$$

For each $n \in \mathbb{N}$ there exists a unique $\left(x_{n}, v_{n}\right)$ solution of the differential inclusion

$$
\begin{aligned}
& v_{n}(t) \in \partial \varphi\left(x_{n}(t)\right), \quad 0 \leq t \leq T, \\
& \lambda_{n}(t) \dot{x}_{n}(t)+\dot{v}_{n}(t)+v_{n}(t)+\nabla \psi\left(x_{n}(t)\right)=0, \\
& \text { a.e. } 0 \leq t \leq T \\
& x_{n}(0)=x_{0}, \quad v_{n}(0)=v_{0} .
\end{aligned}
$$

We will show that $\left(x_{n}, v_{n}\right)$ converges uniformly to a solution of (51-53).

Consider, in $C([0, T], H \times H)$, the norm

$$
\|(z, w)\|_{c_{0}}=\max _{t \in[0, T]} \sqrt{c_{0}^{2}\|z(t)\|^{2}+\|w(t)\|^{2}} .
$$

It is equivalent to the sup norm in $C([0, T], H \times H)$. Using Theorem3.1, (46) and (54) we deduce the existence of a constant $C$ (which is independant of $n$ ) such that for any $n, m$ we have

$$
\begin{aligned}
\left\|\left(x_{n}, v_{n}\right)-\left(x_{m}, v_{m}\right)\right\|_{c_{0}} & \leq C \exp \left(\frac{\left\|\dot{\lambda}_{n}+\dot{\lambda}_{m}\right\|_{L^{1}([0, T])}}{2 c_{0}}+T\left(1+\frac{L_{\psi}}{c_{0}}\right)\right)\left\|\lambda_{n}-\lambda_{m}\right\|_{L^{1}([0, T])} \\
& \leq C \exp \left(\frac{T V(\lambda)}{c_{0}}+T\left(1+\frac{L_{\psi}}{c_{0}}\right)\right)\left\|\lambda_{n}-\lambda_{m}\right\|_{L^{1}([0, T])} .
\end{aligned}
$$


Since the sequence $\left(\lambda_{n}\right)_{n \in \mathbb{N}}$ converges to $\lambda$ in $L^{1}(0, T)$, we deduce that $\left(x_{n}, v_{n}\right)$ is a Cauchy sequence with respect to the sup norm. Therefore, $\left(x_{n}, v_{n}\right)$ converges uniformly to some continuous $(x, v):[0, T] \longrightarrow H \times H$. Since $\nabla \psi$ is continuous we also obtain

$$
\nabla \psi\left(x_{n}\right) \rightarrow \nabla \psi(x) \quad \text { uniformly on }[0, T]
$$

Moreover, Lemma 4.2 ensures that $\lambda_{n} \dot{x}_{n}$ and $\dot{v}_{n}$ converge weakly to $\lambda \dot{x}$ and $\dot{v}$ in $L^{2}(0, T ; H)$. Hence letting $n \rightarrow+\infty$ in (56) gives

$$
\lambda \dot{x}+\dot{v}+v+\nabla \psi(x)=0,
$$

that's (52). Finally, since the graph of $\partial \varphi$ is closed we obtain, for all $t \in[0, T], v(t) \in \partial \varphi(x(t))$ that's $(51)$. Hence $(x, v)$ is a solution of (51)-(53).

Uniqueness: We adapt the proof of [6], using differential and integral calculus for BV functions which involves differential measures.

Define $\lambda^{-}:[0, T] \longmapsto\left[c_{0},+\infty[\right.$ by

$$
\begin{aligned}
\lambda^{-}(0) & =\lambda(0), \\
0 & <t \leq T: \lambda^{-}(t)=\lim _{\varepsilon>0, \varepsilon \rightarrow 0} \lambda(t-\varepsilon) .
\end{aligned}
$$

Let $(x, v),(y, w):[0, T] \rightarrow H \times H$ be two strong solutions of (51)-(52)-(53). Explicitly

$$
\begin{aligned}
& \lambda \dot{x}+\dot{v}+v+\nabla \psi(x)=0 \text { a.e.; } v(t) \in \partial \varphi(x(t)) \forall t ; x(0)=x_{0}, v(0)=v_{0}, \\
& \lambda \dot{y}+\dot{w}+w+\nabla \psi(y)=0 \text { a.e.; } w(t) \in \partial \varphi(y(t)) \forall t ; y(0)=x_{0}, w(0)=v_{0} .
\end{aligned}
$$

Since $\lambda=\lambda^{-}$a.e., we also have

$$
\lambda^{-} \dot{x}+\dot{v}+v+\nabla \psi(x)=0 \text { and } \lambda^{-} \dot{y}+\dot{w}+w+\nabla \psi(y)=0 \text { a.e. }
$$

and consequently

$$
\lambda^{-}(\dot{x}-\dot{y})+(\dot{v}-\dot{w})+v-w+\nabla \psi(x)-\nabla \psi(y)=0 \text { a.e. }
$$

In terms of differential measures on $[0, T]$ we have [19]

$$
d\left[\lambda^{-}(x-y)+(v-w)\right]=\lambda^{-} d(x-y)+(x-y) d \lambda^{-}+d(v-w) .
$$

Integrating the left hand term on $[0, s[$ and taking the initial condition into account, we obtain for $s \in[0, T][?$, Corollary 8.2]

$$
\int_{[0, s[} d\left[\lambda^{-}(x-y)+(v-w)\right]=\lambda^{-}(s)(x(s)-y(s))+(v(s)-w(s)) .
$$

Now integrating the right hand term of (59) on $[0, s)$ and taking (58) into account, we get

$$
\begin{aligned}
\int_{[0, s[}\left[\lambda^{-} d(x-y)+(x-y) d \lambda^{-}+d(v-w)\right] & =\int_{[0, s[}\left[\lambda^{-} d(x-y)+d(v-w)\right]+\int_{[0, s[}(x-y) d \lambda^{-} \\
& =\int_{[0, s[}\left[\lambda^{-}(\dot{x}-\dot{y})+\dot{v}-\dot{w}\right] d t+\int_{[0, s[}(x-y) d \lambda^{-} \\
& =-\int_{[0, s[}[v-w+\nabla \psi(x)-\nabla \psi(y)] d t+\int_{[0, s[}(x-y) d \lambda^{-}
\end{aligned}
$$

From $(59,60,61)$ we deduce

$$
\lambda^{-}(s)(x(s)-y(s))+(v(s)-w(s))=\int_{[0, s[}(x-y) d \lambda^{-}-\int_{[0, s[}[v-w+\nabla \psi(x)-\nabla \psi(y)] d t .
$$

Whence

$\left\|\lambda^{-}(s)(x(s)-y(s))+(v(s)-w(s))\right\| \leq \int_{[0, s[}\|x-y\|\left|d \lambda^{-}\right|+\int_{[0, s[}\|v-w\| d t+\int_{[0, s[}\|\nabla \psi(x)-\nabla \psi(y)\| d t$ 
Define $\theta(s)=\left(c_{0}^{2}\|x(s)-y(s)\|^{2}+\|v(s)-w(s)\|^{2}\right)^{1 / 2}$. The same reasoning as in Theorem 3.1 yields

$\theta(s) \leq\left\|\lambda^{-}(s)(x(s)-y(s))+(v(s)-w(s))\right\|, \forall s \in[0, T]$.

Besides we also have $c_{0}\|x(s)-y(s)\| \leq \theta(s)$, $\|v(s)-w(s)\| \leq \theta(s)$, and we have that $\nabla \psi$ is the gradient of a convex, continuously differentiable function $\psi: H \longrightarrow$ $\mathbb{R}$, and by Lipschitz continuity property of $\nabla \psi$, and definition of $\theta$, we have

$$
\begin{aligned}
\|\nabla \psi(x(t))-\nabla \psi(y(t))\| & \leq L_{\psi}\|x(t)-y(t)\| \\
& \leq \frac{L_{\psi}}{c_{0}} \theta(t) .
\end{aligned}
$$

Hence, with (62)

$$
\begin{aligned}
\theta(s) & \leq \frac{1}{c_{0}} \int_{[0, s[} \theta\left|d \lambda^{-}\right|+\int_{[0, s[} \theta d t \\
& +\frac{1}{c_{0}} L_{\psi} \int_{[0, s[} \theta d t=\int_{[0, s[} \theta d \mu
\end{aligned}
$$

where $d \mu$ denotes the nonnegative measure $\frac{1}{c_{0}}\left|d \lambda^{-}\right|+$ $\left(1+\frac{1}{c_{0}} L_{\psi}\right) d t$

If $\theta \not \equiv 0$ on $[0, T]$, define $t_{0}=\inf \{t \in[0, T], \theta(t)>0\}$. Note $t_{0}<T$ and $\theta\left(t_{0}\right)=0$, since $\theta$ is continuous. With (64) we then have

$$
\theta(s) \leq \int_{] t_{0}, s[} \theta d \mu, t_{0}<s \leq T .
$$

In view of $\int_{\left.] t_{0}, t_{0}\right]} d \mu=0$ and of the right continuity at $t_{0}$ of $t \longrightarrow \int_{\left.] t_{0}, t\right]} d \mu,[?$, Proposition 9.1] there exists some $\left.\left.t_{1} \in\right] t_{0}, T\right]$ such that $\int_{\left.] t_{0}, t_{1}\right]} d \mu<1 / 2$. Let $M$ be an upper bound of $\theta$ on $\left[0, t_{1}\right]$; from (65) we deduce, for $\left.\left.s \in\right] t_{0}, t_{1}\right]$

$$
\theta(s) \leq M \int_{] t_{0}, s[} d \mu \leq M \int_{] t_{0}, t_{1}[} d \mu \leq \frac{M}{2}
$$

Hence $M / 2$ is also an upper bound of $\theta$ on $\left[0, t_{1}\right]$, which necessarily entails $M=0$ and $\theta \equiv 0$ on $\left[0, t_{1}\right]$. But this is contradiction with the definition of $t_{0}$. Hence $\theta \equiv 0$ and $(x, v) \equiv(y, w)$ on $[0, T]$.

Theorem 2.2 has a natural global version formulation:

Corollary 4.1. Suppose that $\lambda:[0, \infty[\rightarrow] 0, \infty[$ is of bounded variation on $[0, T]$ and $\inf \lambda([0, T])>0$ for any $T<\infty$. Let $v_{0} \in \partial \varphi\left(x_{0}\right)$ and $v_{0} \neq 0$. Then there is existence and uniqueness of a strong solution $(x, v):[0, \infty[\longrightarrow H \times H$ of the Cauchy problem

$$
\begin{aligned}
& \lambda \dot{x}+\dot{v}+v+\nabla \psi(x)=0, v(t) \in \partial \varphi(x(t)) \\
& x(0)=x_{0}, v(0)=v_{0}
\end{aligned}
$$

where the first equality holds for almost all $t \in[0, \infty[$, and the inclusion holds for all $t \in[0, \infty[$.

Remark 4.1. The results obtained in this paper still hold if
$B=\nabla \psi$ the gradient of a convex, continuously differentiable function $\psi: H \longrightarrow \mathbb{R}$ is replaced by a maximal monotone cocoercive operator $B: H \longrightarrow H$ (see $[1,4,17,18,21,22]$ ).

\section{Conclusion}

In a Hilbert space setting, we studeid the stability properties of the regularized continuous Newton method with two potentials, which aims at solving inclusions governed by structured monotone operators. The Levenberg-Marquardt regularization term acts in an open loop way. As a byproduct of our study, we took the regularization coefficient of bounded variation. These stability results are directly related to the study of numerical algorithms that combine forward-backward and Newton's methods

Let us list some interesting questions to be examined in the future:

1. Study the asymptotic stability properties, corresponding to the case where $T=+\infty$, in connection with the convergence results of [2] and [7].

2. Extend the results to the case where the LevenbergMarquart regularization term is given in a closed-loop form, $\lambda(t)=\alpha(\|\dot{x}(t)\|)$ as in [6].

3 . Study the same questions for the corresponding forwardbackward algorithms, see [1].

\section{References}

[1] B. Abbas, H. Attouch, Dynamical systems and forward-backward algorithms associated with the sum of a convex subdifferential and a monotone cocoercive operator, Optimization, (2014) http://dx.doi.org/10.1080/02331934.2014.971412.

[2] B. Abbas, H. Attouch, B. F. Svaiter, Newton-like dynamics and forward-backward methods for structured monotone inclusions in Hilbert spaces, J. Optim. Theory Appl., 161 (2014), No. 2, pp. 331-360.

[3] Alvarez, F., Attouch, H., Bolte, J., Redont, P.: A secondorder gradient-like dissipative dynamical system with Hessian-driven damping. Application to optimization and mechanics. J. Math. Pures Appl. 81, 747-779 (2002).

[4] Antipin, A. S.: Minimization of convex functions on convex sets by means of differential equations. Differential Equations. 30, 1365-1375 (1994).

[5] Attouch, H., Briceno, L., Combettes, P.L.: A parallel splitting method for coupled monotone inclusions. SIAM J. Control Optim. 48, 3246-3270 (2010).

[6] H. Attouch, P. Redont, B. F. Svaiter, Global convergence of a closed-loop regularized Newton method for solving monotone inclusions in Hilbert spaces, J. Optim. Theory Appl., 157 (2013), No. 3, pp. 624-650. 
[7] Attouch, H., Svaiter, B.F.: A continuous dynamical Newton-like approach to solving monotone inclusions. SIAM J. Control Optim. 49, 574-598 (2011).

[8] Bauschke, H., Combettes, P.L.: Convex Analysis and Monotone Operator Theory. CMS books in Mathematics, Springer (2011).

[9] Beck, A., Teboulle, M.: Gradient-based algorithms with applications in signal recovery problems. In: Palomar, D., Eldar, Y. (eds.): Convex Optimization in Signal Processing and Communications, pp. 33-88. Cambridge University Press, (2010).

[10] Bolte, J.: Continuous gradient projection method in Hilbert spaces. J. Optim. Theory Appl. 119, 235-259 (2003).

[11] Brézis, H.: Opérateurs Maximaux Monotones et SemiGroupes de Contractions dans les Espaces de Hilbert. North-Holland/Elsevier, New-York (1973).

[12] Brézis, H.: Analyse Fonctionnelle. Masson, Paris (1983).

[13] Bruck, R. E.: Asymptotic convergence of nonlinear contraction semigroups in Hilbert spaces. J. Funct. Anal. 18, 15-26 (1975).

[14] Dennis, J. E., Schnabel, R. B.: Numerical Methods for Unconstrained Minimization. Prentice-Hall, Englewood Cliffs, NJ, 1983. Reprinted by SIAM publications (1993).

[15] Ferreira, O. P., Svaiter, B. F.: Kantorovich's theorem on Newton's method on Riemannian manifolds. Journal of Complexity 18, 304-329 (2002).
[16] Güler, O.: On the convergence of the proximal point algorithm for convex minimization, SIAM J. Control Optim. 29, 403-419 (1991).

[17] Haraux, A.: Systèmes dynamiques dissipatifs et applications. RMA 17, Masson, Paris, (1991).

[18] Nocedal, J., Wright, S.: Numerical Optimization. Springer Series in Operations Research, Springer-Verlag, New-York (1999).

[19] Opial, Z.: Weak convergence of the sequence of successive approximations for nonexpansive mappings. Bull. Amer. Math. Soc. 73, 591-597 (1967).

[20] Peypouquet, J., Sorin, S.: Evolution equations for maximal monotone operators: asymptotic analysis in continuous and discrete time, J. of Convex Analysis 17, 1113-1163 (2010).

[21] Rockafellar, R. T.: Monotone operators and the proximal point algorithm. SIAM J. Control Optim. 14, 877-898 (1976).

[22] Rockafellar, R. T.: Maximal monotone relations and the second derivatives of nonsmooth functions. Ann. Inst. Henri Poincar? 2, 167-184 (1985).

[23] Solodov, M. V., Svaiter, B. F.: A globally convergent inexact Newton method for systems of monotone equations. In: Fukushima, M., Qi, L., (eds): Nonsmooth, Piecewise Smooth, Semismooth and Smoothing Methods, pp. 355-369. Kluwer Academic Publishers, (1999). 